\title{
Trial Production of Variable Pitch Wing Attached Multicopter for Power Saving and Long Flight
}

\author{
Kiyoteru Hayama ${ }^{\mathrm{a},{ }^{*}}$, Hirofumi Ohtsuka ${ }^{\mathrm{b}}$ and Hiroki Irie ${ }^{\mathrm{c}}$ \\ ${ }^{a}$ Dept. of Information, Communication and Electronic Engineering, \\ ${ }^{b}$ Dept. of Control and Information Systems Engineering, National Institute of Technology, Kumamoto College, \\ 2659-2 Suya, Koshi, Kumamoto 861-1102, Japan \\ ${ }^{\mathrm{c}}$ Dept. of Architecture and Civil Engineering, National Institute of Technology, Kumamoto College, \\ 2627, Hirayamashin-machi Yatsushiro, Kumamoto, 866-0074, Japan \\ *hayama@kumamoto-nct.ac.jp
}

\begin{abstract}
The trial production of a new concept vertical take-off and landing (VTOL) aircraft based on variable pitch wing attached multicopter was carried out for aerial, observation and research. Continuous transition from multicopter to similar to fixed-wing aircraft can be done with adjusting suitable angle of attack of the wing of multicopter. The proposed rotorcraft can obtain the lift of wing during horizontal flight, and its causes the reduction of power consumption and extend the flight area. The demonstration experimental rotorcrafts with variable pitch wing multicopter based on the Y3 tricopter and Y6 hexacopter are also presented.
\end{abstract}

Keywords: UAV, VTOL, multicopter, tilt wing, power saving.

\section{Introduction}

The multi-rotor helicopter also called multicopter is widely spread for the less mechanical parts, electrically controllable and small space of the take-off and landing, and is useful for aerial, observation and research applications ${ }^{(1)}$. However, the time-of-flight and cruising distance is limited by the battery capacity. On the other hand, though the fixed-wing aircraft has an advantage of wide range of flight area caused by the high-speed and long-time flight of energy efficiency than the rotorcraft, it cannot hover in the air and takes a runway field to take-off and landing. Assuming the exploration and observation at disaster sites, it is difficult to secure the airfield, therefore, a new flying machine is desired that has both feature of the rotorcraft for vertical take-off and landing (VTOL) and fixed-wing aircraft for wide range of flight. There are many kind of $\mathrm{VTOL}^{(2)}$. It is well known that the tilt rotor system and tail-sitter aircraft. The tail-sitter takes off and land on its tail, then tilts horizontally for forward flight ${ }^{(3)}$. The tilt rotor system can be vertical and horizontal flight by changing the angle of rotors ${ }^{(4)}$. However, attitude control for both aircraft is difficult in transition flight to vertical and horizontal at the time of take-off and landing.

In this study, trial production of a new concept VTOL based on variable pitch wing attached multicopter is presented. Continuous transition from rotorcraft to similar to fixed-wing aircraft can be done with adjusting suitable angle of attack of the wing of multicopter. The proposed rotorcraft can obtain the lift of wing during horizontal flight, and its causes the reduction of power consumption and extend the flight area.

\section{Proposed Rotorcraft}

\subsection{Fixed wing attached multicopter}

Firstly, the most common multicopter, quadcopter is used for the study to investigate the effect of fixed wing attachment. Figure 1 shows the commercially available radio control (R/C) quadcopter TL130H (Tarot) with 15 degrees inclined wing. The wing was placed on the outer side of quadcopter to avoid the turbulence of rotors.

As shown in Fig 2 (a), the quadcopter tilts forward during horizontal flight. Therefore, angle of attack of the inclined wing becomes suitable to generate the lift. As a 


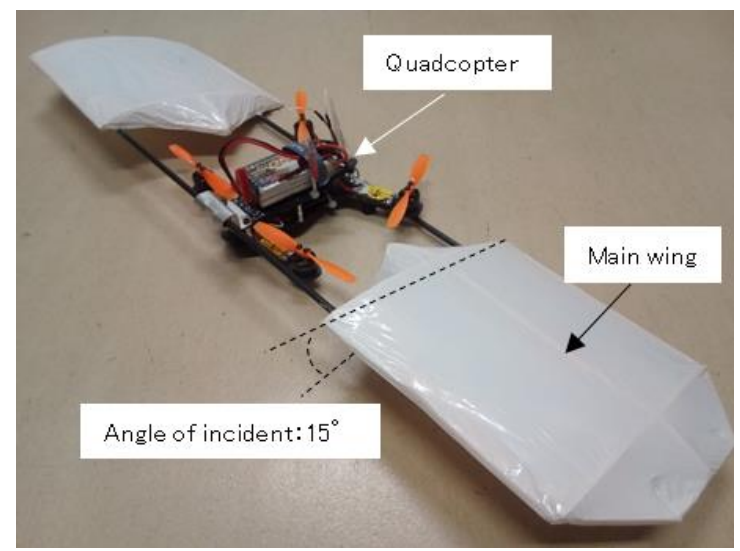

Fig. 1. Fixed wing attached quadcopter.

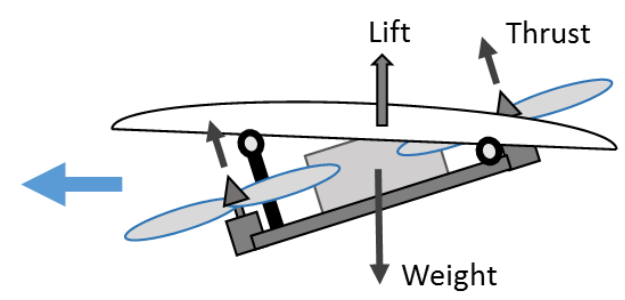

(a) Side view of the quadcopter during horizontal flight

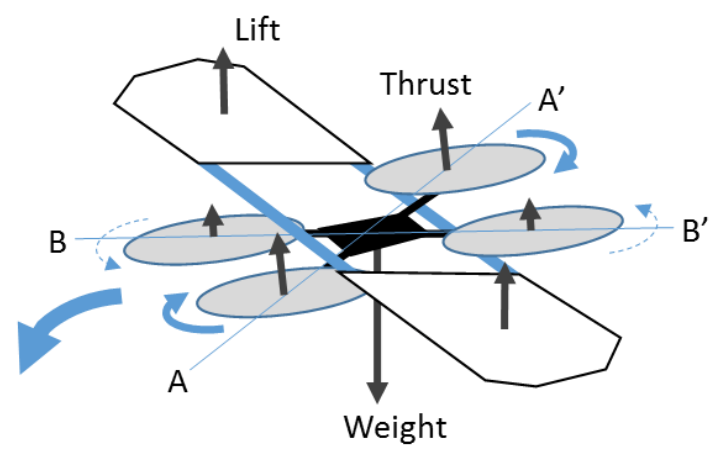

(b) Left turning of the quadcopter

Fig. 2. Schematic of the fixed wing attached

result of the test flight, we have confirmed that stable hovering and transition to horizontal flight are possible. As the flight speed increases, the quadcopter rises due to the lift of wing, the throttle should be down to lower the thrust of rotors to keep the altitude. However, in order to keep constant angle of attack, a fine pitch control was required. Also, this quadcopter lacked stability when turning flight. The reason can be explained by Fig. 2(b) showing left turning. In order to generate a yaw moment, the thrust on the $\mathrm{A}-\mathrm{A}^{\prime}$ axis rotors must be strengthened and the thrust on the $B-B^{\prime}$ axis rotors must be weakened, therefore, it is difficult to stabilize because the thrust of B-B' axis rotors is insufficient to keep the stability around A-A' axis. Moreover, it is difficult to make place of wing on quadcopter.

From these point, we will discuss based on the Y type frame multicopter which can directly generate yaw moment and does not always impair the stability of rotors placed in the plane.

\subsection{Variable pitch wing attached multicopter}

Figure 3 shows the schematic of principle for proposed rotorcraft. When the multicopter flights forward, the output of the front rotors decreases and that for rear rotors increases to incline forward the multicopter as shown in figure 3(a). In case of the wing is attached to the multicopter at a fixed angle, the angle of attack of the wing becomes negative as inclined of the multicopter. An effective lift cannot be obtained at this case (Fig. 3(b)). Therefore, we installed a variable pitch wing to the multicopter, mounted a mechanism to control at constant angle of attack of wing during forward flight (Fig. 3(c)). It is thought that this mechanism makes the lift of wing during forward flight, power saving and extend flight area.

The lift of wing supports only a part of weight of the multicopter. Therefore, the aerodynamic center (ac) of wing is attached corresponding above the gravity center, and the

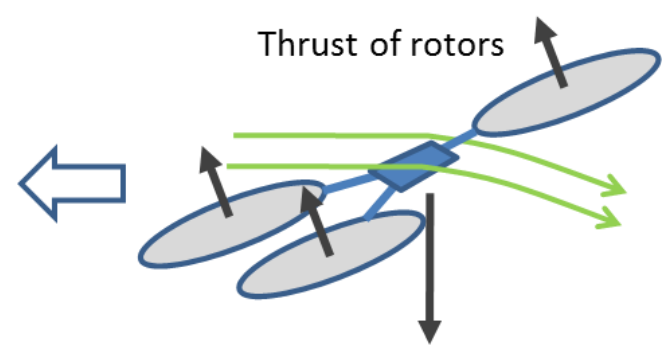

(a) Horizontal flight without wing

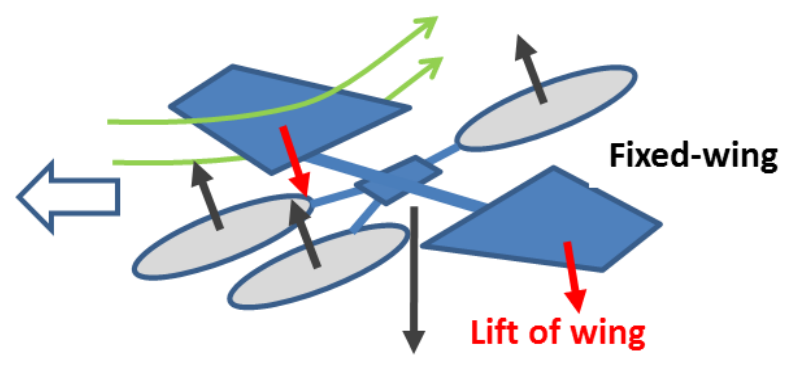

(a) Horizontal flight with fixed-wing

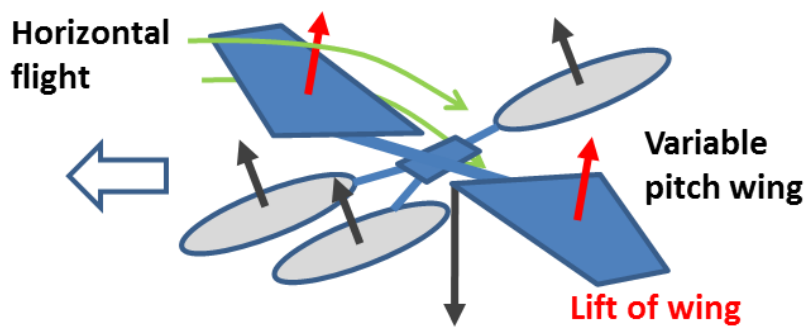

(c) Horizontal flight with variable pitch wing

Fig. 3. Schematic of principle for proposed rotorcraft. 
auxiliary wing such as ailerons is not required.

\subsection{Trial production of the proposed rotorcraft}

Figure 4 shows the photograph of the prototype of the proposed rotorcraft. The base flame is Y3 tricopter, which is made by squared timber mounted three blushless motors. Only tail motor has tilt mechanism with servo linkage for control of yow moment. The maximum thrust force and power consumption of the motor using $8 \times 4$ inches propeller are $0.4 \mathrm{~kg}$ and $80 \mathrm{~W}$, respectively. The airfoil of the wing is an original flat bottom similar to Clark $\mathrm{Y}^{(5)}$. The wing span and width are $0.80 \mathrm{~m}$ and $0.25 \mathrm{~m}$, respectively. The total weight was about $0.73 \mathrm{~kg}$ with LiPo battery $(3 \mathrm{~S}-11.1 \mathrm{~V}$, $1900 \mathrm{mAh}$ ). The wing area is $0.225 \mathrm{~m}^{2}$ and the wing loading is estimated to $27.5 \mathrm{~N} / \mathrm{m}^{2}$. This value is comparable to the commercial electric R/C airplane.

Figure 5 shows the block diagram of the proposed rotorcraft. The rotorcraft is operated by $2.4 \mathrm{GHz} \mathrm{R} / \mathrm{C}$ system. We have used the APM (ArduPilot MEGA 2.5, 3D Robotics $^{(6)}$ ) for attitude control of the rotorcraft. The APM is commercially available flight controller. The control board is developed as open source/hardware project. The 3-axis acceleration sensor, 3-axis gyroscope, 3-axis geomagnetic sensor and pressure sensor are mounted in APM, it is possible to utilize the attitude control that are developed in open source and flight control software (Arducopter). The configuration of the attitude and flight control are also possible by using a ground control software (Mission Planner ${ }^{(7)}$ ). The APM which is installed general tricopter firmware. Stabilization of both hovering and horizontal flight of the proposed rotorcraft is carried out by APM. The APM also has the output for control of camera gimbal, and the output is used for control of pitch angle of the wing.

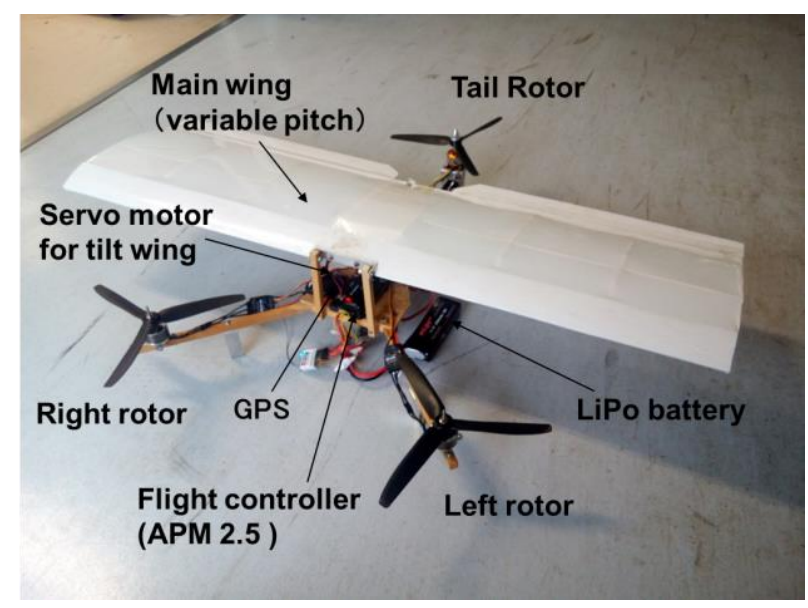

Fig. 4. Trial product of the proposed rotorcraft.

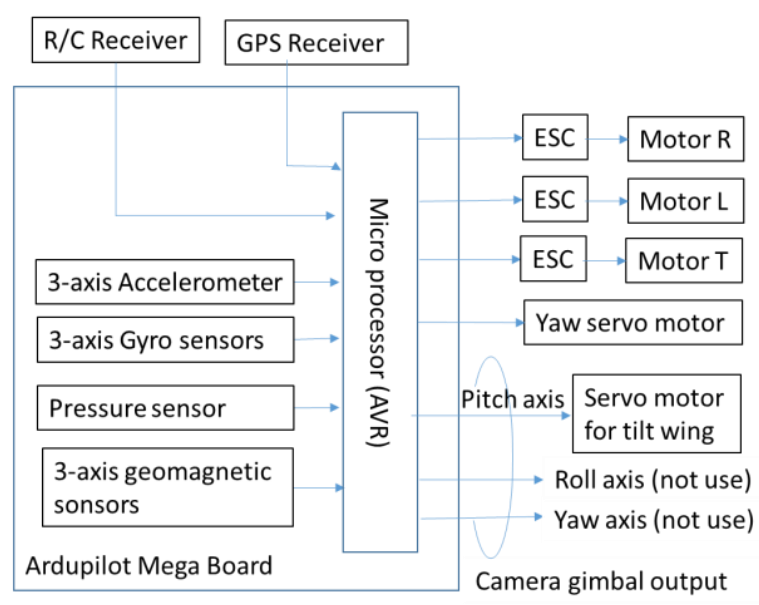

Fig. 5. Block diagram of the proposed rotorcraft.

The linkage mechanism of the wing is shown in Fig. 6. Front edge of the wing is fixed with a hinge, and pitch angle of the wing is controlled by up and down the rear edge with servo motor. Holding torque of the servo motor is $3.2 \mathrm{~kg} \cdot \mathrm{cm}$, which is enough to support the estimated maximum weight of the rear edge of wing. Around $9 \mathrm{~cm}$ link to the rear edge of wing can be supported half of the weight of rotorcraft.

Figure 7 shows a diagram of setting servo parameters for the variable pitch wing. The control signal for pitch of camera gimbal is inverted/non-inverted according to the inclination of pitch of the rotorcraft, adjust the operation rate of the servo motor according to the inclination, and set an arbitrary angle of attack with offset of servo motor. This setting parameters can be done using mission planner.

\section{Test flight}

We conducted a test flight of the prototype of rotorcraft. After take-off the rotorcraft vertically, both body

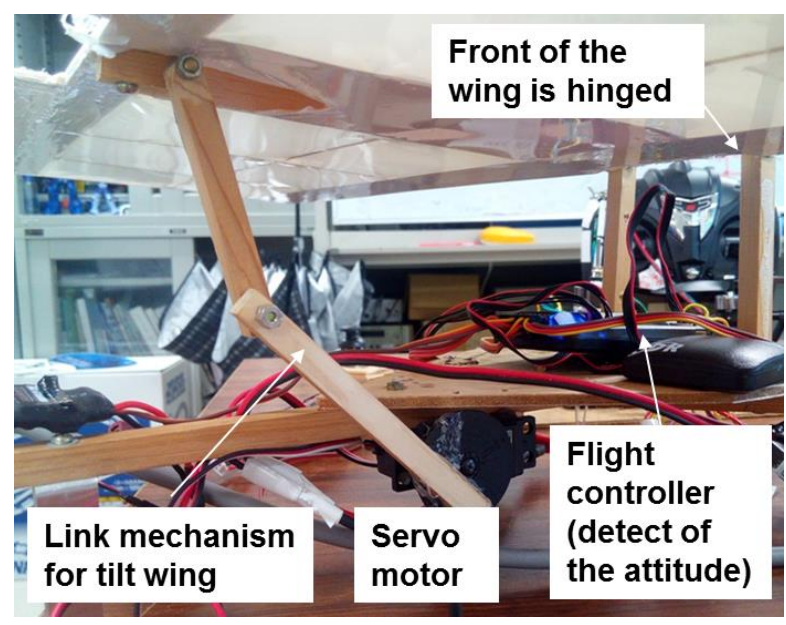

Fig.6. Link mechanism for variable pitch wing. 


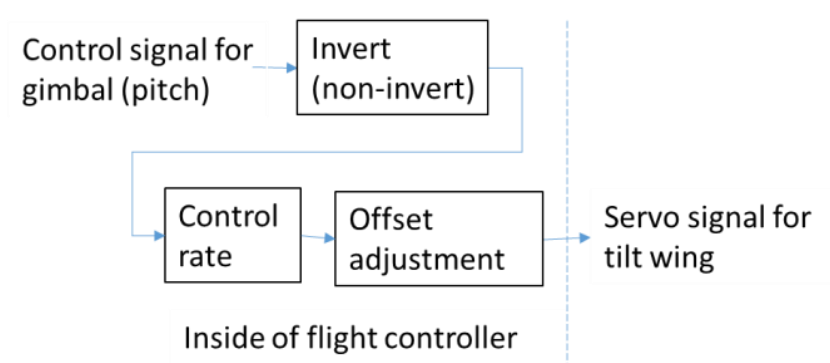

Fig. 7. Diagram of the setting of servo parameters for the variable pitch wing.

and wing kept level and could hover at the place (Fig. 8(a)). Figure 8(b) shows a photograph when the rotorcraft is nose down and flying forward. It can be seen that the wing is substantially horizontal level, though the rotorcraft is nose down.

The power consumption of the rotorcraft with the wing and without the wing (loaded same weight of the wing) is compared. As shown in Table 1, it was possible to fly by $66 \%$ electric power with wing compared with the case without wing. Therefore, the reach distance can be expected about 1.5 times far away.

As the flight speed increases, the wing generates lift

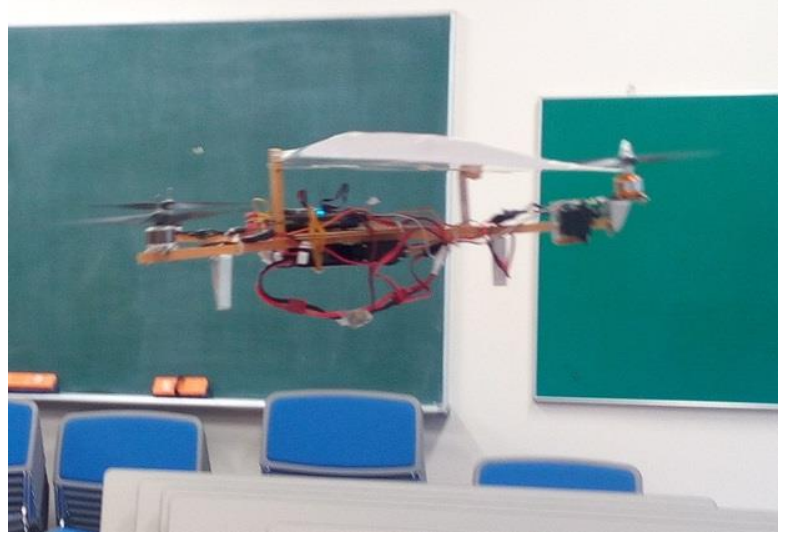

(a) Hovering

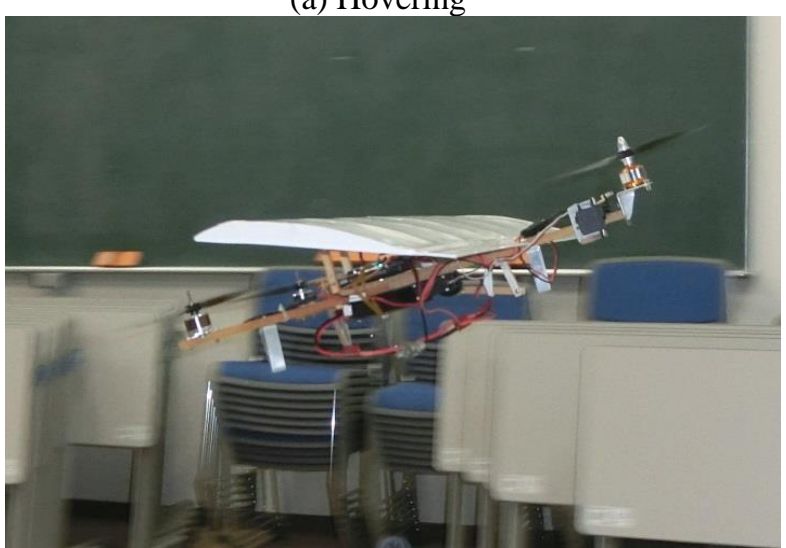

(b) Horizontal flight

Fig.8. Flight image of the proposed rotorcraft.
Table 1. Typical power consumption with and without wing. The battery voltage was $11.1 \mathrm{~V}$ and average flight speed was about $9 \mathrm{~m} / \mathrm{s}$.

\begin{tabular}{|c|c|c|}
\hline Flight condition & Current & Power consumption \\
\hline without wing & $13.6 \mathrm{~A}$ & $151 \mathrm{~W}$ \\
\hline with wing & $9.0 \mathrm{~A}$ & $99 \mathrm{~W}$ \\
\hline
\end{tabular}

supporting the weight of rotorcraft, then the rotorcraft rises the altitude. In order to keep the altitude, it is found that the throttle should to be down, resulting in power saving. Maneuverability is almost same as normal multicopter. There is no discontinuity of maneuver when flight transition from vertical to level flight. Behaviors during backward flight and quick turning is different from the normal multicopter, but it was not to make maneuvering so difficult.

\section{Development of demonstration experimental rotorcraft}

Since it was confirmed that the variable pitch wing attached rotorcraft has advantages in terms of power saving and long flight at straight flight, we have made two types of demonstration experimental rotorcraft that can be used for verification test for civil engineering, surveying and geodetic observation. Figure 9(a) and 9(b) show developed demonstration experimental rotorcraft with variable pitch wing based on Y3 tricopter and Y6 hexacopter, respectively. The motors are arranged in an equilateral triangle at the angle of the motor arms of 120 degrees. The wing shape is sweptback wings which is not interfere with the rotors.

Detailed specification of the rotorcrafts based on the Y3 tricopter and Y6 hexacopter are shown in table 2. Both rotorcrafts have already done the test flight, and their flight performance and stability have been confirmed. But quantitative verification of these rotorcrafts such as power consumption, flight speed, analysis of IMU (inertial measurement unit) signals etc. have not be done.

The estimated flight time from the specification of rotorcrafts and their components is an attractive value for observation surveying operations over a long distance range. Both rotorcrafts have sufficient load capacity, especially the Y6 hexacopter based rotorcraft can be installed high-resolution camera.

In considering the application, for example in a wide range of aerial shots, it is often case that the flight plan is repeat of the straight flight and change direction. The rotorcraft proposed in this study can be saving the power 


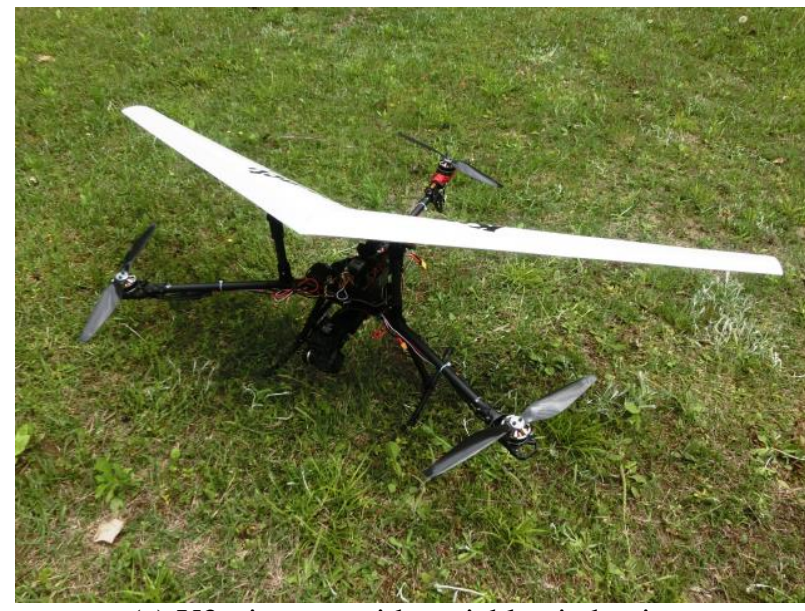

(a) Y3 tricopter with variable pitch wing.

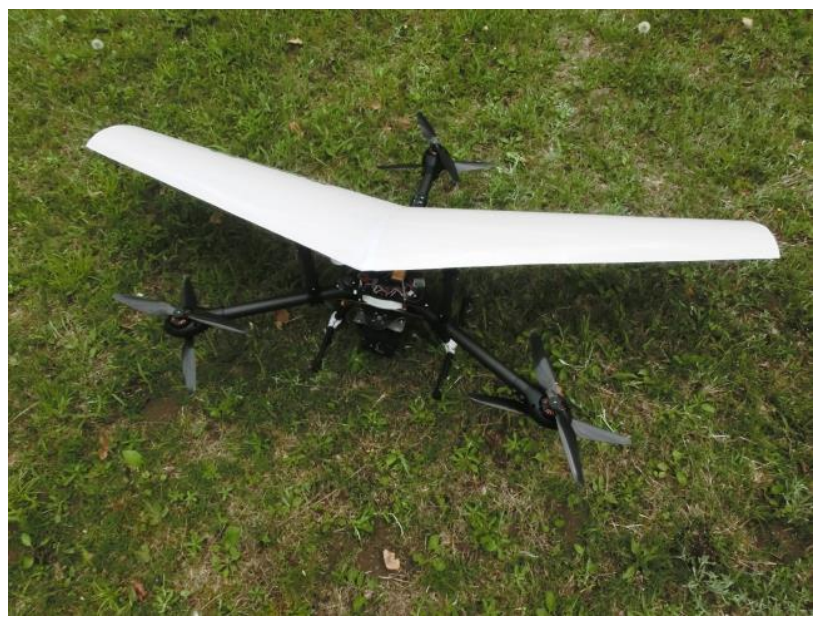

(b) Y6 hexacopter with variable pitch wing.

Fig. 9. Demonstration experimental rotorcrafts with variable pitch wing.

during straight flight, and is advantageous for a wide range observation applications. Three-dimensional visualization of aerial shots has been established which can be easily visualized by commercially available software such as PhotoScan $^{(8)}$.

\section{Conclusions}

Trial production of a new concept VTOL based on variable pitch wing attached multicopter is presented. The reduction of power consumption is observed during straight flight. We have also developed the demonstration experimental rotorcraft based on Y3 tricopter and Y6 hexacopter. More detailed flight data and the advantages for the wide range observation will be demonstrated in near future.
Table 2. Specification of developed demonstration experimental rotorcrafts (estimated values are contained).

\begin{tabular}{|l|c|c|}
\hline Rotorcraft type & Y3 tricopter & Y6 hexacopter \\
\hline Flight controller & APM 2.5 & APM 2.5 \\
\hline Class & $900 \mathrm{~mm}$ & $900 \mathrm{~mm}$ \\
\hline Total weight & $1.8 \mathrm{~kg}$ & $3.8 \mathrm{~kg}$ \\
\hline Motor type & $\begin{array}{c}\text { ARRIS } \\
\text { M3508/580KV }\end{array}$ & $\begin{array}{c}\text { Tarot } \\
4006 / 620 \mathrm{KV}\end{array}$ \\
\hline Motor power & $280 \mathrm{~W}$ & $260 \mathrm{~W}$ \\
\hline Propeller & $14 \times 5 \mathrm{inch}$ & $13 \times 5.5 \mathrm{inch}$ \\
\hline Maximum thrust & $1.8 \mathrm{~kg}$ & $1.6 \mathrm{~kg}$ \\
\hline Number of motor & 3 & 6 \\
\hline Total thrust (est.) & $5.4 \mathrm{~kg}$ & $9.6 \mathrm{~kg}$ \\
\hline Thrust/weight ratio & 2.96 & 2.49 \\
\hline Load capacity (est.) & $3.6 \mathrm{~kg}$ & $5.8 \mathrm{~kg}$ \\
\hline Wing type & original & original \\
\hline Wing span & $1.4 \mathrm{~m}$ & $1.6 \mathrm{~m}$ \\
\hline Wing width (av.) & $0.23 \mathrm{~m}$ & $0.38 \mathrm{~m}$ \\
\hline Wing area & $0.34 \mathrm{~m}^{2}$ & $0.61 \mathrm{~m}^{2}$ \\
\hline Wing load & $52 \mathrm{~N} / \mathrm{m}^{2}$ & $61 \mathrm{~N} / \mathrm{m}^{2}$ \\
\hline Weight of wing & $300 \mathrm{~g}$ & $500 \mathrm{~g}$ \\
\hline Servo type & KRS-788HV & KRS-2552RHV \\
\hline Servo torque & $10.0 \mathrm{kgf} \cdot \mathrm{cm}$ & $14.0 \mathrm{kgf} \cdot \mathrm{cm}$ \\
\hline max. pitch angle & $40 \mathrm{deg}$. & $35 \mathrm{deg}$. \\
\hline Camera & GoPro HERO & $\begin{array}{c}\text { GoPro } \\
\text { SERO3/4 }\end{array}$ \\
\hline Camera gimbal & Tarot TL3T02 & Tarot TL3D02 \\
\hline Test battery & $4 \mathrm{~S}-4800 \mathrm{mAh}$ & $4 \mathrm{~S}-4800 \mathrm{mAh}$ \\
\hline Voltage & $14.8 \mathrm{~V}$ & $14.8 \mathrm{~V}$ \\
\hline $\begin{array}{l}\text { Flight time: } \\
\text { hovering (est.) }\end{array}$ & $25 \mathrm{~min}$ & $12 \mathrm{~min}$ \\
\hline $\begin{array}{l}\text { Flight time: } \\
\text { level (est.) }\end{array}$ & $38 \mathrm{~min}$ & $19 \mathrm{~min}$ \\
\hline & & \\
\hline
\end{tabular}

This work is partially supported by Grant-in-Aid for Scientific Research (C) 17K06952 and for Challenging Exploratory Research 16K14314.

\section{References}

(1) Jan Wendel, Oliver Meister, Christian Schlaile and Gert F. Trommer, "An integrated GPS/MEMS-IMU navigation system for an autonomous helicopter", Aerospace Science and Technology, Vol.10, Issue 6, 2006, pp.527-533.

(2) Koichi Kita, Atsushi Konno, and Masaru Uchiyama," Hovering Control of a Tail-Sitter VTOL Aerial Robot", Journal of Robotics and Mechatronics, Vol.21 No.2 pp. 277-283.

(3) Ryan X-13 Vertijet, (accessed 26 may 2017), https://en.wikipedia.org/wiki/Ryan_X-13_Vertijet

(4) Jeremiah Gertler, "V-22 Osprey Tilt-Rotor Aircraft: Background and Issues for Congress", March 10, 2011, Congressional Research Service Reports, (accessed 26 
may 2017), http://www.fas.org/sgp/crs/weapons/ $\underline{\text { RL31384.pdf }}$

(5) Martin Simons, Model Aeroplane Aerodynamics, Model \& Allied Publications 1978. Chapter 7 - 9, Aerofoil Sections.

(6) 3DR - Drone \& UAV Technology, (accessed 26 may
2017), http://3drobotics.com/

(7) Arducopter | Multicopter UAV, (accessed 26 may 2017), http://copter.ardupilot.com/

(8) Agisoft PhotoScan, (accessed 26 may 2017), http://www.agisoft.com/ 